\title{
Group Theoretical Properties and Band Structure of the Lamé Hamiltonian
}

\author{
Hui LIf, Dimitri KUSNEZOVE, Francesco IACHELLO \\ Center for Theoretical Physics, Sloane Physics Laboratory, \\ Yale University, New Haven, CT 06520-8120
}

December 1999

\begin{abstract}
We study the group theoretical properties of the Lamé equation and its relation to $s u(1,1)$ and $s u(2)$. We compute the band structure, dispersion relation and transfer matrix and discuss the dynamical symmetry limits.
\end{abstract}

PACs numbers: 03.65.Fd, 02.20.-a, 02.20.Sv, 11.30.-j

keywords: representation theory, dynamical symmetry, exactly solvable models, periodic potentials, band structure.

\footnotetext{
${ }^{1}$ email: huili@nst4.physics.yale.edu

${ }^{2}$ email: dimitri@nst4.physics.yale.edu
} 


\section{Introduction}

The use of group theory to study bound and scattering states has found wide application in physics [1]. A common starting point is to identify a spectrum generating algebra or SGA for the problem of interest. This is possible when the Hamiltonian can be expressed in terms of generators of some algebra $G$. Representation theory then can be used to identify exactly solvable limits of the theory, which can then be translated to explicit forms of the Hamiltonian[2]. Connections between bound state or scattering state problems and representations of compact and non-compact groups are well understood. The remaining category, having to do with band structure and periodic potentials, was suggested initially in [3], but remained an open problem until recently. In [4] it was shown that dynamical symmetry techniques and representation theory can be used to solve band structure problems. In particular, for the Scarf Hamiltonian, band structure arises when one uses the complementary series of the projective representations of $s u(1,1)$ (and $s o(2,2)$ for the extended Scarf potential). Further, these dynamical symmetries allowed simple evaluation of the transfer matrix and dispersion relations. In the present study, we consider the band structure problem associated with the Lamé equation, which is not a dynamical symmetry situation, but more generally that of a SGA. We discuss several realizations of the Lamé equation and its relation to $s u(2)$ and $s u(1,1)$, including a discussion of dynamical symmetry limits, generators, dispersion relation and transfer matrix.

While the Lamé equation at first might seem to be an obscure differential equation, it does find surprisingly wide application in physics. For instance, it has been shown that if one uses the periodic potentials in super-symmetric quantum mechanics, the super partners of the Lamé potentials are distinctly different from the original except for $n=1$ which is not self-isospectral. This provides new solvable periodic potentials [5]. The Lamé equation also appears in the topics ranging from solitons to exactly-solvable models. This includes associations with solutions of the periodic KdV equation [6], BPS monopoles[7], sphaleron solutions of the $(1+1)$-dimensional abelian Higgs model [8], sineGordon solitons [9], as well as relations to Calogero-Moser systems [10]. The analysis of this equation has many group theoretical aspects as well. For instance, the Lamé equation can be written in terms of the composition of two first-order matrix operators, where the coefficients of which satisfy so(3) Nahm's equation[11]. It also arises naturally in the context of $s u(2)$ when one tries to separate Laplace's equation in certain coordinate systems 12, 13 as well as in the general classification of Lie algebraic potentials 14] and in quasi-exactly solvable $s l(2)$ models[15]. The discussion of band structure in the context of group theory is more recent however[3], although the system was not solved and the transfer matrix was not determined.

The Jacobian form of the Lamé equation is

$$
-\frac{d^{2}}{d x^{2}}+\kappa^{2} \ell(\ell+1) \operatorname{sn}^{2} x=\mathcal{E} .
$$

We will view this as a Schrödinger equation with mass $M=1 / 2$ when $x$ is real valued. This equation was first studied group theoretically in [13]. The elliptic functions $\operatorname{sn} \alpha=\operatorname{sn}(\alpha \mid \kappa)$, $\operatorname{cn} \alpha=\operatorname{cn}(\alpha \mid \kappa)$, and $\operatorname{dn} \alpha=\operatorname{dn}(\alpha \mid \kappa)$ are doubly-periodic functions in the complex plane, of modulus $\kappa$, where $0 \leq \kappa \leq 1$. We will omit the modulus except in functions where 
it differs from $\kappa$. The complementary modulus is defined as $\kappa^{\prime}=\left(1-\kappa^{2}\right)^{1 / 2}$. The periods of the Jacobi elliptic functions are related to the complete elliptic integrals $K=$ $(\pi / 2) F\left(1 / 2,1 / 2,1 ; \kappa^{2}\right)$ and $K^{\prime}=(\pi / 2) F\left(1 / 2,1 / 2,1 ; \kappa^{\prime 2}\right)$, where $F$ is the hypergeometric function, by:

$$
\begin{array}{llll}
\operatorname{sn}(\alpha+\tau)=\operatorname{sn} \alpha & \tau=2 i K^{\prime}, & 4 K+4 i K^{\prime}, & 4 K \\
\operatorname{cn}(\alpha+\tau)=\operatorname{cn} \alpha & \tau=4 i K^{\prime}, & 2 K+2 i K^{\prime}, & 4 K \\
\operatorname{dn}(\alpha+\tau)=\operatorname{dn} \alpha & \tau=4 i K^{\prime}, & 4 K+4 i K^{\prime}, & 2 K
\end{array}
$$

In the limit $\kappa=1$, the real period related to $K$ becomes infinite, and the functions are no longer periodic on the real axis. Along the real axis, the potential $\operatorname{sn}^{2} x$ is periodic and bounded, while along the imaginary axis, it has periodic singularities of the type $1 / x^{2}$. Examples are shown in Fig. 1 for $\kappa^{2}=1 / 2$ for potentials $V(x)=\operatorname{sn}^{2}(a x+b)$, with several choices of $a$ and $b$.

The Lamé equation has also been related to the band structure problem associated

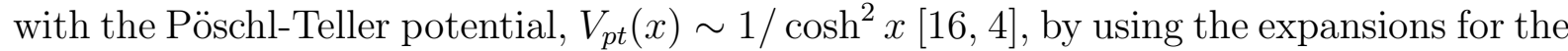
elliptic functions in terms of trigonometric functions found in the exercises of Whittaker and Watson [17]. Specifically, one can start with the 1-d band structure problem

$$
H=-\frac{d^{2}}{d x^{2}}+\sum_{k=-\infty}^{\infty} \frac{g}{\cosh ^{2}\left((x-k a) / x_{0}\right)} .
$$

If we write the coupling constant as

$$
g=-\frac{\ell(\ell+1)}{x_{0}^{2}}
$$

we can make the coordinate transformation

$$
x=i\left(\frac{\pi x_{0}}{2 K}\right) z-\left(\frac{a+i \pi x_{0}}{2}\right)
$$

which transforms the Hamiltonian to the Lamé equation. In this article, we first discuss the relation of su(2) Hamiltonians to the Lamé equation, followed by a discussion of band edges. Next we consider $s u(1,1)$ and the relation to scattering states in the dynamical symmetry limits. Finally we derive the transfer matrix and dispersion relation for the Lamé equation followed by the relation to the group theoretical SGA Hamiltonians.

\section{$2 s u(2)$ Realizations of the Lamé Equation}

\subsection{Sphero-conal Coordinates for $s u(2)$}

In order to realize the Lamé equation from $s u(2)$ or $s u(1,1)$, we will use coordinate systems defined in terms of Jacobi elliptic functions. These are often refered to as sphero-conal 18 or conical 12 coordinates. One can find variations that have been considered in the past, 
and not all provide a good description for all values of the modulus $\kappa$. Consider first the mapping:

$$
x=\kappa \operatorname{sn} \alpha \operatorname{sn} \beta, y=i \frac{\kappa}{\kappa^{\prime}} \operatorname{cn} \alpha \operatorname{cn} \beta, z=\frac{1}{\kappa^{\prime}} \operatorname{dn} \alpha \operatorname{dn} \beta,
$$

The ranges of the angles $\alpha, \beta$ are defined in terms of the elliptic integrals $K$ and $K^{\prime}$, so that they depend on the value of $\kappa$. Specifically $-2 K<\alpha<2 K$ and $K \leq \beta<K+2 i K^{\prime}$ where $\kappa^{\prime 2}=1-\kappa^{2}$, and $x^{2}+y^{2}+z^{2}=1$. The contours of constant $\alpha$ and $\beta$ cover the sphere as illustrated in Fig. 2(a). For $\kappa=1 / 2$, four typical contours (for $\alpha= \pm K / 2$ and $\left.\beta=K+i K^{\prime} / 2, K+3 i K^{\prime} / 2\right)$ are shown in Fig. 2(b). This parametrization can be used to construct the generators of $s u(2)$. A direct computation of the generators yields the realization (I) given in Table 1 . These generators satisfy the commutation relations in the form $\left[L_{a}, L_{b}\right]=\epsilon_{a b c} L_{c}$ with Casimir invariant

$$
\begin{aligned}
C_{2} & =L_{x}^{2}+L_{y}^{2}+L_{z}^{2} \\
& =\frac{1}{\kappa^{2}\left(\operatorname{sn}^{2} \alpha-\operatorname{sn}^{2} \beta\right)}\left(\frac{\partial^{2}}{\partial \alpha^{2}}-\frac{\partial^{2}}{\partial \beta^{2}}\right) .
\end{aligned}
$$

The $s u(2)$ algebras we discuss here have Casimir invariants which have expectation value $-\ell(\ell+1)$. We now consider which types of quadratic Hamiltonians of the form

$$
H=\sum_{j} \eta_{j} L_{j}^{2}
$$

can be constructed which result in Schrödinger equations in $\alpha$ and $\beta$. Specifically, when the coordinate representation of $H$ contains differential opertors only of the type $\partial^{2} / \partial \alpha^{2}$ and $\partial^{2} / \partial \beta^{2}$, one can use $H$ together with $C_{2}$ to perform a separation of variables resulting in two independent Lamé equations. We find the three forms in the top right of Table 1, which are simply related through the Casimir operator. To demonstrate the separation, consider $\mathrm{H}_{3}$,

$$
\begin{aligned}
H_{3} & =L_{z}^{2}+\kappa^{2} L_{y}^{2} \\
& =\frac{1}{\left(\operatorname{sn}^{2} \beta-\operatorname{sn}^{2} \alpha\right)}\left(\operatorname{sn}^{2} \beta \frac{\partial^{2}}{\partial \alpha^{2}}-\operatorname{sn}^{2} \alpha \frac{\partial^{2}}{\partial \beta^{2}}\right),
\end{aligned}
$$

and require it to be a constant of the motion with eigenvalue $H_{3}=\mathcal{E}$. Using basis states denoted by the direct product, $\Psi(\alpha, \beta)=\psi(\alpha) \phi(\beta)$, we arrive at two identical Lamé equations with identical eigenvalue:

$$
\begin{aligned}
{\left[-\frac{d^{2}}{d \alpha^{2}}+\kappa^{2} \ell(\ell+1) \operatorname{sn}^{2} \alpha\right] \psi(\alpha) } & =\mathcal{E} \psi(\alpha), \\
{\left[-\frac{d^{2}}{d \beta^{2}}+\kappa^{2} \ell(\ell+1) \operatorname{sn}^{2} \beta\right] \phi(\beta) } & =\mathcal{E} \phi(\beta) .
\end{aligned}
$$

Thus solution of the eigenvalue problem for $H_{3}=L_{z}^{2}+\kappa^{2} L_{y}^{2}$ yields the single valued solutions to these equations, which will correspond to the band edges. One should keep in mind that while $\alpha$ is defined on the real axis, $\beta$ is complex. Also, while the Hamiltonians $H_{k}$ are well defined in their algebraic form, the generators and coordinates have 
singularities in both $\kappa=0$ and $\kappa=1$ limits. Hence we consider a different realization below.

To reduce these Hamiltonians to more conventional Schrödinger equations in $\alpha$ and $\beta$, we would like the coordinates to be defined along the real axis. To do so, we make a shift (i) $\beta \rightarrow \beta+K+i K^{\prime}$ followed by (ii) $\beta \rightarrow-i \beta$. This results in the transformations:

$$
\begin{aligned}
\operatorname{sn}(\beta \mid \kappa) & \rightarrow \frac{1}{\kappa} \operatorname{dn}\left(\beta \mid \kappa^{\prime}\right), \\
\operatorname{cn}(\beta \mid \kappa) & \rightarrow \frac{\kappa^{\prime}}{i \kappa} \operatorname{cn}\left(\beta \mid \kappa^{\prime}\right), \\
\operatorname{dn}(\beta \mid \kappa) & \rightarrow \kappa^{\prime} \operatorname{sn}\left(\beta \mid \kappa^{\prime}\right) .
\end{aligned}
$$

The new coordinate system is no longer singular, and is parameterized as

$$
x=\operatorname{sn} \alpha \operatorname{dn}\left(\beta \mid \kappa^{\prime}\right), y=\operatorname{cn} \alpha \operatorname{cn}\left(\beta \mid \kappa^{\prime}\right), z=\operatorname{dn} \alpha \operatorname{sn}\left(\beta \mid \kappa^{\prime}\right),
$$

where $-2 K<\alpha<2 K,-K^{\prime} \leq \beta<K^{\prime}$ are now both real valued. Contours of constant $\alpha$ and $\beta$ are shown in Fig. 2(c) for $\alpha= \pm K / 2$ and $\beta= \pm K^{\prime} / 2$. If we construct the generators of $s u(2)$ in this basis, we obtain the realization (II) given in Table 1 . The Casimir invariant now has the form

$$
C_{2}=L_{x}^{2}+L_{y}^{2}+L_{z}^{2}=\frac{-1}{\kappa^{2} \mathrm{cn}^{2} \alpha+\kappa^{\prime 2} \mathrm{cn}^{2}\left(\beta \mid \kappa^{\prime}\right)}\left(\frac{\partial^{2}}{\partial \alpha^{2}}+\frac{\partial^{2}}{\partial \beta^{2}}\right)
$$

The quadratic Hamiltonians which give rise to Schrödinger equations when combined with $C_{2}$ are given in the lower right of Table 1 . Consider again $H_{3}=L_{z}^{2}+\kappa^{2} L_{y}^{2}$. If we set $H_{3}=\mathcal{E}$ and $C_{2}=-\ell(\ell+1)$, we obtain

$$
\begin{aligned}
{\left[-\frac{d^{2}}{d \alpha^{2}}+\kappa^{2} \ell(\ell+1) \operatorname{sn}^{2} \alpha\right] \psi(\alpha) } & =\mathcal{E} \psi(\alpha), \\
{\left[-\frac{d^{2}}{d \beta^{2}}+\kappa^{\prime 2} \ell(\ell+1) \operatorname{sn}^{2}\left(\beta \mid \kappa^{\prime}\right)\right] \phi(\beta) } & =(\ell(\ell+1)-\mathcal{E}) \phi(\beta) .
\end{aligned}
$$

In this case the coordinates are both real valued. For integer $\ell$, and both potentials are non-negative, we see that $0 \leq \mathcal{E} \leq \ell(\ell+1)$. Further the ground state of one Hamiltonian corresponds the the highest energy state of the other, and vice-versa. (Similar equations are obtained if we use $H_{2}$ or $H_{1}$.) Before we discuss some of the general properties of these equations, we consider first the dynamical symmetry limits which are when $\kappa=0$ or 1 .

\section{$2.2 \kappa=0$ Limit}

In the limit $\kappa=0$, and $H_{3}=L_{z}^{2}=m^{2}$, so that $\mathcal{E}=m^{2}$, and the coordinate Hamiltonians are:

$$
\begin{aligned}
-\frac{d^{2}}{d \alpha^{2}} \psi(\alpha) & =\mathcal{E} \psi(\alpha), \\
{\left[-\frac{d^{2}}{d \beta^{2}}-\frac{\ell(\ell+1)}{\cosh ^{2} \beta}\right] \phi(\beta) } & =-\mathcal{E} \phi(\beta) .
\end{aligned}
$$


The generators in this limit have the simpler form:

$$
\begin{aligned}
I_{ \pm} & =e^{ \pm i \alpha}\left[\mp \cosh \beta \frac{\partial}{\partial \beta}+i \sinh \beta \frac{\partial}{\partial \alpha}\right] \\
I_{3} & =-i \frac{\partial}{\partial \alpha}
\end{aligned}
$$

The first equation is free motion, while the second corresponds to bound states of the Pöschl-Teller potential. For the discrete representations of $s u(2)$, one obtains the bound state spectrum $-\mathcal{E}=-m^{2}$. The wavefunctions are of the form:

$$
\Psi(\alpha, \beta ; \kappa=0) \sim P_{\ell}^{m}(\tanh \beta) \exp ( \pm i m \alpha), \quad m=-\ell, \ldots, \ell ; \quad \ell=0,1,2, \ldots
$$

with $-\pi<\alpha<\pi,-\infty<\beta<\infty$.

\section{$2.3 \kappa=1$ Limit}

In the limit $\kappa=1, H_{3}=L^{2}-L_{x}^{2}$, the equations reduce to

$$
\begin{aligned}
{\left[-\frac{d^{2}}{d \alpha^{2}}-\frac{\ell(\ell+1)}{\cosh ^{2} \alpha}\right] \psi(\alpha) } & =[\mathcal{E}-\ell(\ell+1)] \psi(\alpha)=\mathcal{E}^{\prime} \psi(\alpha) \\
-\frac{d^{2}}{d \beta^{2}} \phi(\beta) & =[\ell(\ell+1)-\mathcal{E}] \phi(\beta)=-\mathcal{E}^{\prime} \phi(\beta)
\end{aligned}
$$

The generators are the same as in the $\kappa=0$ limit, with $\alpha$ and $\beta$ interchanged. The second equation is now free motion, while the first corresponds to bound states of the PöschlTeller potential with a shifted eigenvalue. For the discrete representations of $s u(2)$, one obtains the bound state spectrum $\mathcal{E}^{\prime}=-m^{2}$. The wavefunctions are of the form:

$$
\Psi(\alpha, \beta ; \kappa=1) \sim P_{\ell}^{m}(\tanh \alpha) \exp ( \pm i m \beta), \quad m=-\ell, \ldots, \ell ; \quad \ell=0,1,2, \ldots
$$

with $-\infty<\alpha<\infty,-\pi<\beta<\pi$.

\subsection{Band Edges and $s u(2)$}

For general values of $\kappa$, the Lamé Hamiltonians are periodic (as seen in Fig. 1), and will have band structure. As the discrete representations of $s u(2)$ correspond to single-valued wavefunctions, the discrete representations can at most describe the band edges. Since we have an algebraic realization of the Hamiltonians whose spectrum corresponds to the Lamé equations, we can obtain the eigenvalues of the Hamiltonian $H_{k}$ (and hence the band edges) by a direct diagonalization in the spherical harmonic basis $|\ell m\rangle$, which can then be related to the results for the elliptic basis through the coordinate transformations. The resulting functions are doubly periodic solutions of Lamé's equation known as Lamé polynomials [18]. These polynomials are of the form $\operatorname{sn}^{a} x \operatorname{cn}^{b} x \operatorname{dn}^{c} x F_{p}\left(\operatorname{sn}^{2} x\right)$, where $a, b, c=$ 0,1 and $a+b+c+2 p=\ell$. Here $F_{p}(z)$ is a polynomial in $z$ of order $p$. A discussion of 
these functions can be found in [19, 17, 18]. For $\ell=1$, there are three eigenstates of $H_{3}$ given by

$$
\begin{aligned}
& \Psi_{1}(\alpha, \beta ; \kappa)=|10\rangle \sim \operatorname{sn} \alpha \operatorname{dn}\left(\beta \mid \kappa^{\prime}\right) \\
& \Psi_{2}(\alpha, \beta ; \kappa)=\frac{1}{\sqrt{2}}[|11\rangle+|1-1\rangle] \sim \operatorname{cn} \alpha \operatorname{cn}\left(\beta \mid \kappa^{\prime}\right) \\
& \Psi_{3}(\alpha, \beta ; \kappa)=\frac{1}{\sqrt{2}}[|11\rangle-|1-1\rangle] \sim \operatorname{dn} \alpha \operatorname{sn}\left(\beta \mid \kappa^{\prime}\right)
\end{aligned}
$$

with eigenvalues $E_{1}=1+\kappa^{2}, E_{2}=1, E_{3}=\kappa^{2}$. In the dynamical symmetry limits, these three states reduce to those discussed above.

For $\ell=2$, we have

$$
\begin{aligned}
\Psi_{1}(\alpha, \beta ; \kappa) & =\frac{1}{\sqrt{2}}[|22\rangle-|2-2\rangle] \sim \operatorname{cn} \alpha \operatorname{dn} \alpha \operatorname{sn}\left(\beta \mid \kappa^{\prime}\right) \operatorname{cn}\left(\beta \mid \kappa^{\prime}\right) \\
\Psi_{2}(\alpha, \beta ; \kappa) & =\frac{1}{\sqrt{2}}[|21\rangle+|2-1\rangle] \sim \operatorname{sn} \alpha \operatorname{cn} \alpha \operatorname{cn}\left(\beta \mid \kappa^{\prime}\right) \operatorname{dn}\left(\beta \mid \kappa^{\prime}\right) \\
\Psi_{3}(\alpha, \beta ; \kappa) & =\frac{1}{\sqrt{2}}[|21\rangle-|2-1\rangle] \sim \operatorname{sn} \alpha \operatorname{dn} \alpha \operatorname{sn}\left(\beta \mid \kappa^{\prime}\right) \operatorname{dn}\left(\beta \mid \kappa^{\prime}\right) \\
\Psi_{4}(\alpha, \beta ; \kappa) & \sim|22\rangle+|2-2\rangle+\sqrt{\frac{2}{3}} \frac{2 f_{+}(\kappa)-1-\kappa^{2}}{1-\kappa^{2}}|20\rangle \\
& \sim\left(1-f_{+}(\kappa) \operatorname{sn}^{2} \alpha\right)\left(1-f_{-}\left(\kappa^{\prime}\right) \operatorname{sn}^{2}\left(\beta \mid \kappa^{\prime}\right)\right) \\
\Psi_{5}(\alpha, \beta ; \kappa) & \sim|22\rangle+|2-2\rangle+\sqrt{\frac{2}{3}} \frac{2 f_{-}(\kappa)-1-\kappa^{2}}{1-\kappa^{2}}|20\rangle \\
& \sim\left(1-f_{-}(\kappa) \operatorname{sn}^{2} \alpha\right)\left(1-f_{+}\left(\kappa^{\prime}\right) \operatorname{sn}^{2}\left(\beta \mid \kappa^{\prime}\right)\right)
\end{aligned}
$$

with eigenvalues

$$
\begin{aligned}
& E_{1}=1+\kappa^{2}, \quad E_{2}=4+\kappa^{2}, \quad E_{3}=1+4 \kappa^{2}, \\
& E_{4}=2 f_{+}(\kappa), \quad E_{5}=2 f_{-}(\kappa),
\end{aligned}
$$

where $f_{ \pm}(\kappa)=\left(1+\kappa^{2} \pm \sqrt{1-\kappa^{2} \kappa^{\prime 2}}\right)$. In general there will be a relation between the eigenstates $|\ell m\rangle$ of the $2 \ell+1$ band edges and products of Lamé polynomials. The band edges for $\ell=1,2$ are shown in Fig. 3 as a function of $\kappa^{2}$. The bands are indicated by the shaded regions. The dashed line indicates the height of the potential. One can see that the bands merge at $\kappa=0$, and pass to the bound and scattering states of the Pöschl-Teller potential for $\kappa=1$.

\section{$3 \mathrm{su}(1,1)$ Realizations of the Lamé Equation}

\subsection{Elliptic Parametrization of $\operatorname{su}(1,1)$}

In order to discuss the eigenstates in the band, and develop the dispersion relation, we require more general representations. In the spirit of recent work on the Scarf potential 
where it was shown that one can use a $s u(1,1)$ dynamical symmetry to analytically solve for the dispersion relation[四], states and transfer matrix, we consider transforming our generators to $s u(1,1)$. Consider the transformation of our previous coordinates associated with $x \rightarrow-i x, y \rightarrow-i y:$

$$
x=-i \kappa \operatorname{sn} \alpha \operatorname{sn} \beta, y=\frac{\kappa}{\kappa^{\prime}} \operatorname{cn} \alpha \operatorname{cn} \beta, z=\frac{1}{\kappa^{\prime}} \operatorname{dn} \alpha \operatorname{dn} \beta,
$$

where $0 \leq \alpha<4 K, 0 \leq \beta<i K^{\prime}[13]$. This now parametrizes the hyperbolic surface $z^{2}-x^{2}-y^{2}=1$ illustrated in Fig. 4(a) for $\kappa^{2}=1 / 2$. Contours are shown for selected values of $\alpha$ and $\beta$ in Fig. 4(b). The generators of $s u(1,1)$ algebra are given in Table 2 as realization $(\mathrm{I})$, and satisfy the commutation relations $\left[L_{x}, L_{y}\right]=-L_{z},\left[L_{y}, L_{z}\right]=L_{x}$, and $\left[L_{z}, L_{x}\right]=L_{y}$. The Casimir invariant now has the form

$$
C_{2}=L_{z}^{2}-L_{x}^{2}-L_{y}^{2}=\frac{-1}{\kappa^{2}\left(\operatorname{sn}^{2} \alpha-\operatorname{sn}^{2} \beta\right)}\left(\frac{\partial^{2}}{\partial \alpha^{2}}-\frac{\partial^{2}}{\partial \beta^{2}}\right)
$$

As in the $s u(2)$ case, there are three forms of bilinear Hamiltonians which lead to Schrödinger equations in $\alpha$ and $\beta$. These are given in the top right of Table 2. If we choose $H_{3}=L_{z}^{2}-\kappa^{2} L_{x}^{2}=\mathcal{E}$, together with the Casimir invariant $C_{2}=-\ell(\ell+1)$, we obtain the decoupled Lamé equations:

$$
\begin{aligned}
& {\left[-\frac{d^{2}}{d \alpha^{2}}+\kappa^{2} \ell(\ell+1) \operatorname{sn}^{2} \alpha\right] \psi(\alpha)=-\mathcal{E} \psi(\alpha),} \\
& {\left[-\frac{d^{2}}{d \beta^{2}}+\kappa^{2} \ell(\ell+1) \operatorname{sn}^{2} \beta\right] \phi(\beta)=-\mathcal{E} \phi(\beta) .}
\end{aligned}
$$

Again similar results are obtained if we use $H_{1}$ or $H_{2}$, the only difference arising in the definition of the eigenvalue. Note that the range of $\beta$ is along the imaginary axis. This realization is problematic in the $\kappa=0$ and $\kappa=1$ limits since the generators become singular. Consequently we consider a slightly different realization of $s u(1,1)$ below.

\subsection{Another su(1,1) Realization}

While we do not have a coordinate system which avoids the singularities at $\kappa=1$, it is possible to at least allow a study of the scattering states when $\kappa=0$. To do this we make the transformations (i) $\beta \rightarrow \beta+K+i K^{\prime}$ followed by (ii) $\beta \rightarrow-i \beta$. As a result:

$$
\begin{aligned}
\operatorname{sn}(\beta \mid \kappa) & \rightarrow \frac{1}{\kappa} \operatorname{dn}\left(\beta \mid \kappa^{\prime}\right), \\
\operatorname{cn}(\beta \mid \kappa) & \rightarrow \frac{\kappa^{\prime}}{i \kappa} \operatorname{cn}\left(\beta \mid \kappa^{\prime}\right), \\
\operatorname{dn}(\beta \mid \kappa) & \rightarrow \kappa^{\prime} \operatorname{sn}\left(\beta \mid \kappa^{\prime}\right) .
\end{aligned}
$$

This results in coordinates 13

$$
x=-i \operatorname{sn} \alpha \operatorname{dn}\left(\beta \mid \kappa^{\prime}\right), y=-i \operatorname{cn} \alpha \operatorname{cn}\left(\beta \mid \kappa^{\prime}\right), z=\operatorname{dn} \alpha \operatorname{sn}\left(\beta \mid \kappa^{\prime}\right) \text {, }
$$


where $0 \leq \alpha<4 K,-i K \leq \beta<-i K+K^{\prime}$, also satisfying $z^{2}-x^{2}-y^{2}=1$. Typical contours for this parametrization are shown in Fig. 4(c). The generators are given in Table 2 as realization (II) and satisfy $\left[L_{x}, L_{y}\right]=-L_{z},\left[L_{y}, L_{z}\right]=L_{x}$, and $\left[L_{z}, L_{x}\right]=L_{y}$ with Casimir invariant

$$
C=L_{z}^{2}-L_{x}^{2}-L_{y}^{2}=\frac{1}{\kappa^{2} \operatorname{cn}^{2} \alpha+\kappa^{\prime 2} \operatorname{cn}^{2}\left(\beta \mid \kappa^{\prime}\right)}\left(\frac{\partial^{2}}{\partial \alpha^{2}}+\frac{\partial^{2}}{\partial \beta^{2}}\right)
$$

The Hamiltonians which result in seperable Hamiltonians are given in the bottom right of Table 2. Using $H_{1}=L_{x}^{2}+\kappa^{\prime 2} L_{y}^{2}=\mathcal{E}$ and $C_{2}=-\ell(\ell+1)$ leads to the two Lamé equations:

$$
\begin{aligned}
{\left[-\frac{d^{2}}{d \alpha^{2}}+\kappa^{2} \ell(\ell+1) \operatorname{sn}^{2} \alpha\right] \psi(\alpha) } & =\mathcal{E} \psi(\alpha) \\
{\left[-\frac{d^{2}}{d \beta^{2}}+\kappa^{\prime 2} \ell(\ell+1) \operatorname{sn}^{2}\left(\beta \mid \kappa^{\prime}\right)\right] \phi(\beta) } & =(\ell(\ell+1)-\mathcal{E}) \phi(\beta) .
\end{aligned}
$$

It should be kept in mind that $\beta$ is still complex valued. Never the less this realization allows an analysis of the $\kappa=0$ dynamical symmetry.

\section{$3.3 \kappa=0$ Limit}

This realization is not singular in the $\kappa=0$ limit (although it is in the $\kappa=1$ case). Taking $\kappa=0$, and shifting $\beta$ to be on the real axis by $\beta=\theta-i \pi / 2$, we find

$$
\begin{aligned}
L_{ \pm} & = \pm e^{ \pm i \alpha}\left(\mp \sinh \theta \frac{\partial}{\partial \theta}+i \cosh \theta \frac{\partial}{\partial \alpha}\right) \\
L_{z} & =-\frac{\partial}{\partial \alpha}
\end{aligned}
$$

To recover the usual $s u(1,1)$ commutation relations $\left[I_{z}, I_{ \pm}\right]= \pm I_{ \pm},\left[I_{+}, I_{-}\right]=-2 I_{z}$, we then identify $I_{ \pm}= \pm L_{ \pm}$and $I_{z}=i L_{z}$. It is convient to perform the transformations: $\theta \rightarrow \theta-i \pi / 2, \tanh \theta \rightarrow \cos \theta$, followed by a similarity transformation $f(\theta)=\sqrt{\sin \theta}$, and $\theta \rightarrow i \theta$. Then we have the form:

$$
\begin{aligned}
-\frac{d^{2}}{d \alpha^{2}} \psi(\alpha) & =m^{2} \psi(\alpha), \\
{\left[-\frac{d^{2}}{d \theta^{2}}+\frac{m^{2}-1 / 4}{\sinh ^{2} \theta}\right] \phi(\theta) } & =-\left(\ell+\frac{1}{2}\right)^{2} \phi(\theta) .
\end{aligned}
$$

The principal series $\ell=-1 / 2+i \rho$ of the projective representations of $s u(1,1)$ now describe these scattering states, and the eigenfunctions are of the form:

$$
\Psi_{\ell}^{m} \sim \sqrt{i \sinh \theta} P_{\ell}^{m}(\cosh \theta) e^{ \pm i m \alpha} \quad m \in \Re, \ell=-1 / 2+i \rho, \rho>0 .
$$




\section{Band Structure of the Lamé Hamiltonian}

We now focus on the properties of the Lamé equation in the form of Eq. (1). As we are interested in band structure, the eigenstates must satisfy Bloch's theorem. For a potential which is periodic with period $a, V(x+a)=V(x)$, the wavefunctions must be of the form

$$
\Psi_{k}(x)=u_{k}(x) \exp [-i k x]
$$

where $k$ is the wavenumber and $u_{k}(x)$ has the periodicity of the lattice: $u_{k}(x+a)=u_{k}(x)$. As the eigenstates $\Psi_{k}(x)$ are not periodic, the doubly periodic solutions of the Lamé equation do not play a role for energies in the band. Rather, we look to the more general class of solutions expressed in terms of Jacobi theta functions [17]. Starting with the Hamiltonian

$$
H \psi=\left[-\frac{d^{2}}{d x^{2}}+\kappa^{2} \ell(\ell+1) \operatorname{sn}^{2}(x \mid \kappa)\right] \psi(x)=\mathcal{E} \psi(x),
$$

the solutions for positive integer $\ell$ are given parametrically by

$$
\psi(x)=\prod_{n=1}^{\ell}\left[\frac{\mathcal{H}\left(x+\alpha_{n}\right)}{\theta(x)} e^{-x Z\left(\alpha_{n}\right)}\right]
$$

where $\mathcal{H}$ and $\theta$ are theta functions, and $\alpha_{1}, \alpha_{2}, \ldots \alpha_{\ell}$ are constants determined by the constraints:

$$
\begin{aligned}
\mathcal{E} & =\sum_{n=1}^{\ell} \frac{1}{\operatorname{sn}^{2} \alpha_{n}}-\left[\sum_{n=1}^{\ell} \operatorname{cn} \alpha_{n} \operatorname{dn} \alpha_{n} / \operatorname{sn} \alpha_{n}\right]^{2} \\
0 & =\sum_{p=1}^{\ell} \frac{\operatorname{sn} \alpha_{p} \operatorname{cn} \alpha_{p} \operatorname{dn} \alpha_{p}+\operatorname{sn} \alpha_{n} \operatorname{cn} \alpha_{n} \operatorname{dn} \alpha_{n}}{\operatorname{sn}^{2} \alpha_{p}-\operatorname{sn}^{2} \alpha_{n}} \quad(p \neq n)
\end{aligned}
$$

If this solution is not doubly periodic, a second solution is

$$
\psi^{\prime}(x)=\prod_{n=1}^{\ell}\left[\frac{\mathcal{H}\left(x-\alpha_{n}\right)}{\theta(x)} e^{x Z\left(\alpha_{n}\right)}\right] .
$$

We can then identify the dispersion relation by putting these wavefunctions in Bloch form and by using the periodicity of the theta functions to extract $u_{k}(x)$. We find

$$
k(\mathcal{E})=-i \sum_{n=1}^{\ell} Z\left(\alpha_{n} \mid \kappa^{2}\right)+\frac{\ell \pi}{2 K} .
$$

We will start with the case of $\ell=1$, where simple anlytic results can be obtained. We then derive the transfer matrix for the general case of integer $\ell$.

\section{1 $\ell=1$ Results}

Since there is only one parameter $\alpha$, the constraint equation is simply

$$
\operatorname{dn}^{2} \alpha=\mathcal{E}-\kappa^{2} .
$$


The condition that the dispersion relation is real, $\operatorname{Re} Z\left(\alpha \mid \kappa^{2}\right)=0$, results in two energy bands given by

$$
\kappa^{2} \leq \mathcal{E} \leq 1, \quad 1+\kappa^{2} \leq \mathcal{E}
$$

These are shown in Fig. 3 (top). In the lower band, $\alpha$ has the form $\alpha=K+i \eta$, where $\eta$ ranges from $K^{\prime}$ at $\mathcal{E}=\kappa^{2}$, to 0 at $\mathcal{E}=1$. In the upper band, $\alpha=i \eta$, where $\eta$ ranges from 0 at $\mathcal{E}=1+\kappa^{2}$, to $K^{\prime}$ as $\mathcal{E} \rightarrow \infty$. This path traced out by the parameter $\alpha$ as a function energy $\mathcal{E}$ is shown schematically in Fig. 5. The upper and lower sides correspond to band gaps while the right and left edges are the energy bands. Using the specific forms of $\alpha$ for each band, the dispersion relation becomes

$$
k(\mathcal{E})= \begin{cases}-Z\left(\eta \mid \kappa^{\prime 2}\right)+\frac{\pi}{2 K}\left(1-\frac{\eta}{K^{\prime}}\right)+\sqrt{\frac{\left(\mathcal{E}-\kappa^{2}\right)(1-\mathcal{E})}{1+\kappa^{2}-\mathcal{E}}} & \kappa^{2} \leq \mathcal{E} \leq 1 \\ -Z\left(\eta \mid \kappa^{\prime 2}\right)+\frac{\pi}{2 K}\left(1-\frac{\eta}{K^{\prime}}\right)+\sqrt{\frac{\left(\mathcal{E}-\kappa^{2}-1\right)\left(\mathcal{E}-\kappa^{2}\right)}{\mathcal{E}-1}} & 1+\kappa^{2} \leq \mathcal{E}\end{cases}
$$

This is plotted in Fig. 6 (a) for the case of $\ell=1$. The momentum $k$ is plotted up to the edge of the Brillouin zone, which is $k=\pi / 2 K$. In the figure we use $\kappa^{2}=1 / 2$, so that the band edges are $\mathcal{E}=1 / 2,1$ and $3 / 2$. (The analogous behavior for $\ell=2$ is indicated in Fig. 6(b).) The solution of the Lamé equation in Bloch form is now

$$
\psi_{k}(x)=\left\{\begin{array}{ll}
{\left[\frac{H_{1}(x+i \eta)}{\Theta(x)} \exp (i \pi x / 2 K)\right] \exp (-i k x)} & \kappa^{2} \leq \mathcal{E} \leq 1 \\
{\left[\frac{H(x+i \eta)}{\Theta(x)} \exp (i \pi x / 2 K)\right] \exp (-i k x)} & 1+\kappa^{2} \leq \mathcal{E}
\end{array} .\right.
$$

The component of the wavefunction in square brackets can be checked to be periodic with the periodicity of the direct lattice: $x \rightarrow x+2 K$.

The dispersion relation displays the desired limits. One can see that $k(\mathcal{E}) \rightarrow 0$ as $\mathcal{E} \rightarrow \kappa^{2}$, and $k(\mathcal{E}) \rightarrow \pi / 2 K$ as $\mathcal{E} \rightarrow 1$. Further, as the modulus of the elliptic function vanishes, $\kappa^{2} \rightarrow 0$, the Hamiltonian becomes that of a free system, and we find $k(\mathcal{E}) \rightarrow \sqrt{\mathcal{E}}$ as desired. In the Pöschl-Teller limit, $\kappa^{2} \rightarrow 1$, the band vanishes, and we find $k(\mathcal{E}) \rightarrow 0$. Similar results hold for the upper band as well.

From the dispersion relation we can compute the group velocity and effective mass. To do so, we use the relation between the zeta function and the elliptic integrals of the first and second kind

$$
Z(\alpha)=E(\alpha)-\frac{E\left(\kappa^{2}\right)}{K} \alpha
$$

where $E\left(\kappa^{2}\right)=\frac{\pi}{2} F\left(-1 / 2,1 / 2 ; 1 ; \kappa^{2}\right)$ and $K=\frac{\pi}{2} F\left(1 / 2,1 / 2 ; 1 ; \kappa^{2}\right)$ are the complete elliptic integrals and $E(\alpha)$ is incomplete. Then, the group velocity is given by:

$$
\frac{1}{\nu}=\frac{d k(\mathcal{E})}{d \mathcal{E}}
$$

or

$$
\nu(\mathcal{E})=\frac{2 \sqrt{(1-\mathcal{E})\left(\mathcal{E}-\kappa^{2}\right)\left(1+\kappa^{2}-\mathcal{E}\right)}}{\kappa^{2}+\frac{E\left(\kappa^{2}\right)}{K}-\mathcal{E}} .
$$

We plot $\nu^{2}$ as a function of energy in Fig. 7 for several values of $\kappa$. As $\kappa$ approaches zero, the energy gaps vanish, and the group velocity approaches the free particle limit 
$E=M \nu^{2} / 2=\nu^{2} / 4$ (dot-dashed line). As $\kappa$ approaches unity, the lower band vanishes becoming a bound state, and the group velocity is only non-vanishing for the continuum states of the Pöschl-Teller potential with $\mathcal{E} \geq 2$.

The effective mass $M^{*}$ is determined by

$$
\begin{aligned}
\frac{1}{M^{*}}= & \frac{d \nu}{d k} \\
= & -2 \frac{\left(\mathcal{E}-\kappa^{2}\right)\left(1+\kappa^{2}-\mathcal{E}\right)+(\mathcal{E}-1)\left(1+\kappa^{2}-\mathcal{E}\right)-(\mathcal{E}-1)\left(\mathcal{E}-\kappa^{2}\right)}{\left(\mathcal{E}-\kappa^{2}-\frac{E\left(\kappa^{2}\right)}{K}\right)^{2}} \\
& +4 \frac{(\mathcal{E}-1)\left(\mathcal{E}-\kappa^{2}\right)\left(1+\kappa^{2}-\mathcal{E}\right)}{\left(\mathcal{E}-\kappa^{2}-\frac{E\left(\kappa^{2}\right)}{K}\right)^{3}}
\end{aligned}
$$

We plot $1 / M^{*}$ in Fig. 8 for selected values of $\kappa$. One can see that as $\kappa^{2} \rightarrow 0$, the gaps vanish and $M^{*} \rightarrow M=1 / 2$ as expected for the free particle.

\subsection{Transfer Matrix for the Lamé Hamiltonian}

The general form of the transfer matrix is computed using the definitions in the Appendix. Using the wavefunctions and Eq. (A1), we have

$$
r=-\sum_{n=1}^{\ell}\left[Z\left(\alpha_{n}\right)-\frac{\operatorname{sn} \alpha_{n} \operatorname{dn} \alpha_{n}}{\operatorname{cn} \alpha_{n}}\right]-i \frac{\ell \pi}{2 k} .
$$

Consequently, we see that

$$
r+i k(\mathcal{E})=\sum_{n=1}^{\ell} \frac{\operatorname{sn} \alpha_{n} \operatorname{dn} \alpha_{n}}{\operatorname{cn} \alpha_{n}} .
$$

The transfer matrix then has the form

$$
T=\left(\begin{array}{cc}
\cos 2 k(\mathcal{E}) K & i\left(\sum_{n=1}^{\ell} \frac{\operatorname{sn} \alpha_{n} \operatorname{dn} \alpha_{n}}{\operatorname{cn} \alpha_{n}}\right)^{-1} \sin 2 k(\mathcal{E}) K \\
i\left(\sum_{n=1}^{\ell} \frac{\operatorname{sn} \alpha_{n} \operatorname{dn} \alpha_{n}}{\operatorname{cn} \alpha_{n}}\right) \sin 2 k(\mathcal{E}) K & \cos 2 k(\mathcal{E}) K
\end{array}\right) .
$$

(Note that this is in the form of Eq. (A.3) rather than (A.5).) While this expression requires knowledge of the parameters $\alpha_{n}$, one can obtain various limits of this for the free particle and Pöschl-Teller potentials.

\subsection{The $\kappa=0$ Free Particle Limit}

We start first with the $\ell=1$ case. Taking $\kappa=0$ in our transfer matrix, we obtain for the upper and lower bands

$$
T=\left(\begin{array}{cc}
\cosh 2 k(\mathcal{E}) K & i \frac{\operatorname{cn} \alpha}{\operatorname{sn} \alpha \operatorname{dn} \alpha} \sinh 2 k(\mathcal{E}) K \\
i \frac{\sin \alpha \operatorname{dn} \alpha}{\operatorname{cn} \alpha} \sinh 2 k(\mathcal{E}) K & \cosh 2 k(\mathcal{E}) K
\end{array}\right) .
$$


According to Eq. (40),

$$
\frac{\operatorname{sn} \alpha \operatorname{dn} \alpha}{\operatorname{cn} \alpha}=\left[\frac{\left(1+\kappa^{2}-\mathcal{E}\right)\left(\mathcal{E}-\kappa^{2}\right)}{(\mathcal{E}-1)}\right]^{1 / 2}
$$

Taking $\kappa=0$, we have $r \rightarrow 0$, so that

$$
k(\mathcal{E})=-i \frac{\operatorname{sn} \alpha \operatorname{dn} \alpha}{\operatorname{cn} \alpha}=\sqrt{\mathcal{E}} .
$$

The transfer matrix becomes that for a free particle, given by:

$$
T=\left(\begin{array}{cc}
\cos \pi \sqrt{\mathcal{E}} & \mathcal{E}^{-1 / 2} \sin \pi \sqrt{\mathcal{E}} \\
-\mathcal{E}^{1 / 2} \sin \pi \sqrt{\mathcal{E}} & \cos \pi \sqrt{\mathcal{E}}
\end{array}\right)
$$

For the general case of integer $\ell$, we start with the constraint equations (40) and take the limit $\kappa \rightarrow 0$. We first must show that

$$
r+i k(\mathcal{E})=\sum_{n=1}^{\ell} \tan \alpha_{n} \rightarrow i \sqrt{\mathcal{E}}
$$

Then, from the definition of $r$ in the appendix, it must vanish for free motion, so that $r \rightarrow 0$ implies $k(\mathcal{E}) \rightarrow \sqrt{\mathcal{E}}$. Then we recover the free particle transfer matrix. While we can show that the sum in Eq. (59) tends to $i \sqrt{\mathcal{E}}$ for small $\ell$ on a case by case basis, we do not yet have a general proof. However, since the transfer matrix must be that of a free particle in this limit, it is clear that Eq. (59) must hold, and we can use this instead to provide an additional relation among the parameters $\alpha_{n}$.

\subsection{The $\kappa=1$ Pöschl-Teller Limit}

In the limit $\kappa=1$, the Hamiltonian $H$ becomes the Pöschl-Teller Hamiltonian, and our transfer matrix should reduce to that case. We start first with $\ell=1$ and examine the upper band.(The lower band becomes degenerate at $\kappa=1$ ). In this limit, $K \rightarrow \infty$ and $K^{\prime} \rightarrow \pi / 2$. For the upper band,

$$
k(\mathcal{E}) \rightarrow \sqrt{\mathcal{E}-2}+\frac{\pi+2 i \alpha}{2 K}
$$

From Eq. (56), we have in the $\kappa=1$ limit:

$$
\frac{\operatorname{sn} \alpha \operatorname{dn} \alpha}{\operatorname{cn} \alpha}=\tanh \alpha=i \sqrt{\mathcal{E}-2}
$$

The asymptotic form of the transfer matrix $($ as $K \rightarrow \infty)$ becomes:

$$
T=\left(\begin{array}{cc}
\cos (2 K \sqrt{\mathcal{E}-2}+2 i \alpha) & (\mathcal{E}-2)^{-1 / 2} \sin (2 K \sqrt{\mathcal{E}-2}+2 i \alpha) \\
-(\mathcal{E}-2)^{1 / 2} \sin (2 K \sqrt{\mathcal{E}-2}+2 i \alpha) & \cos (2 K \sqrt{\mathcal{E}-2}+2 i \alpha)
\end{array}\right)
$$


We must now change this form of the transfer matrix, defined for periodic potentials $T$, to the form used in the Pöschl-Teller case which is not periodic, $\mathcal{T}$ (see Appendix). Using $k(\mathcal{E})=\sqrt{\mathcal{E}-2}$, we find:

$$
\mathcal{T}_{\kappa=1}=\left(\begin{array}{cc}
\frac{\Gamma(i k) \Gamma(1+i k)}{\Gamma(2+i k) \Gamma(i k-1)} & 0 \\
0 & \frac{\Gamma(-i k) \Gamma(1-i k)}{\Gamma(2-i k) \Gamma(-i k-1)}
\end{array}\right)
$$

For the case of arbitrary $\ell$, we have the more general relations:

$$
\sum_{n=1}^{\ell} \frac{\operatorname{sn} \alpha_{n} \operatorname{cn} \alpha_{n}}{\operatorname{dn} \alpha_{n}} \rightarrow \sum_{n=1}^{\ell} \tanh \alpha_{n}=i \sqrt{\mathcal{E}-\ell(\ell+1)}
$$

The dispersion relation becomes

$$
k(\mathcal{E}) \rightarrow-i \sum_{n=1}^{\ell}\left(\tanh \alpha_{n}-\frac{\alpha_{n}}{K}\right)+\ell \frac{\pi}{2 K}=\sqrt{\mathcal{E}-\ell(\ell+1)}+\ell \frac{\pi}{2 K}+i \frac{1}{K} \sum_{n=1}^{\ell} \alpha_{n}
$$

To compute the form of the transfer matrix for the Pöschl-Teller potential, we use the notation

$$
\mathcal{T}=\left(\begin{array}{cc}
F & G \\
G^{*} & F^{*}
\end{array}\right)
$$

where, from the appendix, we have:

$$
F=(-)^{\ell} \exp \left[-2 \sum_{n=1}^{\ell} \alpha_{n}\right]=\prod_{n=1}^{\ell} \frac{\tanh \alpha_{n}-1}{\tanh \alpha_{n}+1}
$$

This can be further simplified using the limiting form of the constraint equations (40):

$$
\begin{aligned}
\mathcal{E} & =-\left[\sum_{n=1}^{\ell} \frac{1-\tanh ^{2} \alpha_{n}}{\tanh \alpha_{n}}\right]^{2}+\sum_{n=1}^{\ell} \operatorname{coth}^{2} \alpha_{n} \\
0 & =\sum_{p=1(p \neq n)}^{\ell} \frac{\left(1-\tanh ^{2} \alpha_{n}\right) \tanh \alpha_{n}+\left(1-\tanh ^{2} \alpha_{p}\right) \tanh \alpha_{p}}{\tanh ^{2} \alpha_{p}-\tanh ^{2} \alpha_{n}}
\end{aligned}
$$

For small $\ell(\ell=1,2)$ we can solve these equations to show that

$$
F=\frac{\Gamma(i k) \Gamma(1+i k)}{\Gamma(1+i k+\ell) \Gamma(i k-\ell)}, \quad G=0 .
$$

This reduces to the correct form of the transfer matrix. For arbitrary $\ell$, we have not been able to solve these equations, but the result must still hold, since it is governed by the form of the Hamiltonian. Again, we can use these relations to provide additional relations between the parameters $\alpha_{n}$. 


\section{Conclusions}

We have presented a group theoretical analysis of the Lamé equation, which is an example of a SGA band structure problem for $s u(2)$ and $s u(1,1)$. We have computed the dispersion relation and transfer matrix, and discussed the limiting dynamical symmetry limits of these results which correspond to the Pöschl-Teller and free particle Hamiltonians. Because the general Hamiltonian is not of the dynamical symmetry the spectrum cannot be obtained in closed form. Never the less, a diagonalization of Hamiltonians which are bilinear in the angular momentum generators will provide the general solution. There are still many open questions associtated with the group theoretical treatment of the Lamé equation. It would be nice to develop a $s u(1,1)$ parametrization which is non-singular for all values of $\kappa$. In addition, the Scarf and Mathieu equation limits would be interesting to realize more expliticly in the transfer matrix and dispersion relations. Finally, the diagonalization of the algebraic Hamiltonians in the continuum $s u(1,1)$ bases would be interesting to study. It is clear that the result of the diagonalization must yield the same transcendental equations for the parameters $\alpha_{n}$, but their origin would be different.

\section{Appendix: Form of the transfer matrix for periodic potentials}

For symmetric periodic potentials with period $\tau$, we can derive a general formular of the transfer matrix. Suppose for a specific energy E, we have two bloch solutions $u_{+}(x) e^{i k x}$, $u_{-}(x) e^{-i k x}$, where $x$ is the coordinate, $k=k(\mathcal{E})$ is the dispersion relation. Since the potential is symmetric, we can define 21]

$$
r=\frac{u_{+}^{\prime}(\tau / 2)}{u_{+}(\tau / 2)}=-\frac{u_{-}^{\prime}(\tau / 2)}{u_{-}(\tau / 2)} .
$$

Then the transfer matrix is

$$
T=\left(\begin{array}{cc}
\cos k \tau & \frac{i}{i k+r} \sin k \tau \\
i(i k+r) \sin k \tau & \cos k \tau
\end{array}\right) .
$$

The forms of transfer matrices used for periodic and non-periodic potentials is different. In the discussion of the Lamé equation, the $\kappa \rightarrow 1$ limit takes a periodic potential to a non-periodic one, so that we require the transformations that take us from one standard form to the other. If we express the transfer matrix $T$ for the periodic potential as

$$
T=\left(\begin{array}{cc}
\operatorname{Re}(F \exp (i k \tau)+G) & \frac{1}{k} \operatorname{Im}(F \exp (i k \tau)+G) \\
k \operatorname{Im}(F \exp (i k \tau)-G) & \operatorname{Re}(F \exp (i k \tau)-G)
\end{array}\right),
$$

then that of the non-periodic limit will have the form

$$
\mathcal{T}=\left(\begin{array}{cc}
F & G \\
G^{*} & F^{*}
\end{array}\right) .
$$


In this notation, $k=k(\mathcal{E})$ is the dispersion relation, and $\tau$ is the period of the periodic potential which tends to $\infty$. 


\section{References}

[1] For a survey, see for example: F. Iachello, Rev. Nuovo Cimento 191 (1996), and references there in; Dynamical Groups and Spectrum Generating Algebras, Eds. A.Barut, A. Bohm and Y.Ne'eman (World Scientific, Singapore, 1987); F. Iachello and R. Levine, Algebraic Theory of Molecules (Oxford Press, Oxford, 1995); F. Iachello and A. Arima, The Interacting Boson Model.

[2] D. Kusnezov, Phys. Rev. Lett. 79537 (1997).

[3] Y. Alhassid, F. Gürsey and F. Iachello, Phys. Rev. Lett. 50, 873 (1983); F. Gürsey, in Group Theoretical Methods in Physics XI (Springer-Verlag, Berlin, 1983) p.106.

[4] H. Li and D. Kusnezov, Phys. Rev. Lett. 831283 (1999); H. Li and D. Kusnezov, preprint (1999).

[5] A. Khare and U. Sukhatme, quant-ph/9906044; G. Dunne and J. Mannix, Phys. Lett.B428, 115 (1998); G. Dunne and J. Feinberg, Phys. Rev.D57, 1271 (1998).

[6] S. Novikov, S.V. Manakov, L.P. Pitaevskii and V.E. Zakharov, Theory of Solitons, (New York, Plenum, 1984);

[7] P.M. Sutcliffe,J. Phys.A29, 5187 (1996).

[8] Y. Brihaye, S. Giller, P. Kosinski and J. Kunz, Phys. Lett.B293, 383 (1992).

[9] J. Liang, H.J.W. Muller-Kirsten and D.H. Tchrakian, Phys. Lett.B282, 105 (1992).

[10] V.I. Enolskii and J.C. Eilbeck, J. Phys. A28, 1069(1995); J. Dittrich and V.I. Inozemtsev, J. Phys. A26, L753 (1993).

[11] R. S. Ward, J. Phys. A20, 2679 (1987).

[12] W. Miller, Symmetry and Separation of Variables, (Addison-Wesley, Reading, MA, 1977).

[13] J. Patera and P. Winternitz, J. Math. Phys. 14, 1130 (1973); N.W. Macfadyen and P. Winternitz, J. Math. Phys. 12, 281(1971); E. Kalnins and W. Miller Jr., J. Math. Phys. 15, 1263 (1974).

[14] N. Kamran and P.J. Olver, J. Math. Anal. Appl. 145, 342 (1990).

[15] A. V. Turbiner, J. Phys. A22, L1 (1989).

[16] B. Sutherland, Phys. Rev. A 8, 2514 (1973).

[17] E.T. Whittaker and G.N. Watson, A Course of Modern Analysis, (Cambridge Univ. Press, Cambridge, 1980).

[18] F. M. Arscott, Periodic Differential Equations, (Pergamon, Oxford, 1981). 
[19] A. Erdélyi et al, Higher Transcedental Functions, Vol. 3, (McGraw-Hill, New York, 1953).

[20] Y. Alhassid, F. Gürsey, F. Iachello, Ann. Phys. (NY) 148, 346 (1983); Ann. Phys. (NY) 167, 181 (1983).

[21] H.M. James, Phys. Rev. 76, 1602 (1949). 


\section{Figure Captions}

Figure 1. Various forms of the Lamé potential which are real valued. We plot $V(x)$ for modulus $\kappa^{2}=1 / 2$ given by $(i) \operatorname{sn}^{2}(x \mid \kappa)$ (solid), (ii) $\operatorname{sn}^{2}\left(x+i K^{\prime} \mid \kappa\right)(\operatorname{dots}),\left(\right.$ iii) $\operatorname{sn}^{2}(x+K+$ $\left.i K^{\prime} \mid \kappa\right)$ (dashes), (iv) $\operatorname{sn}^{2}(i x \mid \kappa)$ (dot-dashes). For other values of $x$, the potential is periodic, but generally complex.

Figure 2. Coordinate systems for $s u(2)$. (a) Parametrization of the sphere. (b) Contours of constant $\alpha$ and $\beta$. (c) Same as (b) but for the second parametrization.

Figure 3. Band edges from the $s u(2)$ realization of the Lamé equation as a function of $\kappa^{2}$ for $\ell=1$ (top) and $\ell=2$ (bottom). The eigenvalues are indicated. The bands are given by the shaded regions. The dashed line indicates the value of the energy which is at the maximum of the potential $V(x)=\kappa^{2} \ell(\ell+1) \operatorname{sn}^{2} x$

Figure 4. Coordinate systems for $s u(1,1)$. (a) Parametrization of the hyperboloid. (b) Contours of constant $\alpha$ and $\beta$. (c) Same as (b) but for the second parametrization.

Figure 5. Evolution of the parameter $\alpha$ for the $\ell=1$ Lamé equation. The lower energy band has parameter $\alpha=K+i \eta$ where $\eta$ ranges from $K^{\prime}$ at the lower end to 0 at the upper end. The valence band starts with $\alpha=0$ and grows to $\alpha=i K^{\prime}$ as the energy $E \rightarrow \infty$.

Figure 6. (a) Dispersion relation $\mathcal{E}(k)$ for the $\ell=1$ Lamé equation with $\kappa^{2}=1 / 2$. The momentum is plotted in units of $\pi / 2 K$. (b) Analogous behavior for the $\ell=2$ case.

Figure 7. Group velocity for the $\ell=1$ Lamé equation for several values of the modulus $\kappa$. We show $v^{2}$ as a function of energy $\mathcal{E}$. When $\kappa=0$, the Hamiltonian is that of a free particle so $\mathcal{E} \propto v^{2}$ (dot-dashes). As $\kappa$ increases to unity, the lower energy band shrinks to a single bound state of multiplity 2 corresponding to the Pöschl-Teller potential, and the group velocity is only non-zero in the continuum, $\mathcal{E}>2$.

Figure 8. Behavior of $1 / M^{*}$ as a function of energy $\mathcal{E}$ for $\ell=1$ Lamé equation, where $M^{*}$ is the effective mass. As $\kappa \rightarrow 0$, the gap closes, and $M^{*} \rightarrow M=1 / 2$ for this Hamiltonian. 
Table 1. Realizations of $S U(2)$ with $\left[L_{i}, L_{j}\right]=\epsilon_{i j k} L_{k}$ and corresponding Hamiltonians which lead to the Lamé equation.

\section{$S U(2)$ Realization}

(I)

$$
\begin{aligned}
L_{x} & =i\left[\kappa\left(\operatorname{sn}^{2} \alpha-\operatorname{sn}^{2} \beta\right)\right]^{-1} \\
& \times\left[\operatorname{sn} \alpha \operatorname{cn} \beta \operatorname{dn} \beta \partial_{\alpha}-\operatorname{cn} \alpha \operatorname{dn} \alpha \operatorname{sn} \beta \partial_{\beta}\right] \\
L_{y} \quad & =\left[\kappa \kappa^{\prime}\left(\operatorname{sn}^{2} \alpha-\operatorname{sn}^{2} \beta\right)\right]^{-1} \\
& \times\left[\operatorname{cn} \alpha \operatorname{sn} \beta \operatorname{dn} \beta \partial_{\alpha}-\operatorname{sn} \alpha \operatorname{dn} \alpha \operatorname{cn} \beta \partial_{\beta}\right] \\
L_{z} \quad & =i\left[\kappa^{\prime}\left(\operatorname{sn}^{2} \alpha-\operatorname{sn}^{2} \beta\right)\right]^{-1} \\
& \times\left[\operatorname{dn} \alpha \operatorname{sn} \beta \operatorname{cn} \beta \partial_{\alpha}-\operatorname{sn} \alpha \operatorname{cn} \alpha \operatorname{dn} \beta \partial_{\beta}\right]
\end{aligned}
$$

$$
C_{2}=L_{x}^{2}+L_{y}^{2}+L_{z}^{2}=\left[\kappa^{2}\left(\operatorname{sn}^{2} \beta-\operatorname{sn}^{2} \alpha\right)\right]^{-1}\left(\partial_{\alpha}^{2}-\partial_{\beta}^{2}\right)
$$

\section{(II):}

$$
\begin{aligned}
L_{x} & =\left[\kappa^{2} \operatorname{cn}^{2} \alpha+\kappa^{\prime 2} \operatorname{cn}^{2}\left(\beta \mid \kappa^{\prime}\right)\right]^{-1} \\
& \times\left[\kappa^{\prime 2} \operatorname{sn} \alpha \operatorname{sn}\left(\beta \mid \kappa^{\prime}\right) \operatorname{cn}\left(\beta \mid \kappa^{\prime}\right) \partial_{\alpha}+\operatorname{cn} \alpha \operatorname{dn} \alpha \operatorname{dn}\left(\beta \mid \kappa^{\prime}\right) \partial_{\beta}\right] \\
L_{y} & =\left[\kappa^{2} \operatorname{cn}^{2} \alpha+\kappa^{\prime 2} \operatorname{cn}^{2}\left(\beta \mid \kappa^{\prime}\right)\right]^{-1} \\
& \times\left[-\operatorname{cn} \alpha \operatorname{sn}\left(\beta \mid \kappa^{\prime}\right) \operatorname{dn}\left(\beta \mid \kappa^{\prime}\right) \partial_{\alpha}+\operatorname{sn} \alpha \operatorname{dn} \alpha \operatorname{cn}\left(\beta \mid \kappa^{\prime}\right) \partial \beta\right] \\
L_{z} \quad & =\left[\kappa^{2} \operatorname{cn}^{2} \alpha+\kappa^{\prime 2} \operatorname{cn}^{2}\left(\beta \mid \kappa^{\prime}\right)\right]^{-1} \\
& \times\left[-\operatorname{dn} \alpha \operatorname{cn}\left(\beta \mid \kappa^{\prime}\right) \operatorname{dn}\left(\beta \mid \kappa^{\prime}\right) \partial_{\alpha}-\kappa^{2} \operatorname{sn} \alpha \operatorname{cn} \alpha \operatorname{sn}\left(\beta \mid \kappa^{\prime}\right) \partial_{\beta}\right] \\
C_{2} & =L_{x}^{2}+L_{y}^{2}+L_{z}^{2} \\
& =\left[\kappa^{2} \operatorname{cn}^{2} \alpha+\kappa^{\prime 2} \operatorname{cn}^{2}\left(\beta \mid \kappa^{\prime}\right)\right]^{-1}\left(\partial_{\alpha}^{2}+\partial_{\beta}^{2}\right)
\end{aligned}
$$

$$
\begin{aligned}
H_{1} & =L_{x}^{2}+\kappa^{\prime 2} L_{y}^{2} \\
& =\left[\kappa^{2}\left(\operatorname{sn}^{2} \alpha-\mathrm{sn}^{2} \beta\right)\right]^{-1}\left(\operatorname{dn}^{2} \beta \partial_{\alpha}^{2}-\mathrm{dn}^{2} \alpha \partial_{\beta}^{2}\right) \\
H_{2} & =\kappa^{2} L_{x}^{2}-\kappa^{\prime 2} L_{z}^{2} \\
& =\left[\operatorname{sn}^{2} \beta-\operatorname{sn}^{2} \alpha\right]^{-1}\left(\mathrm{cn}^{2} \beta \partial_{\alpha}^{2}-\mathrm{cn}^{2} \alpha \partial_{\beta}^{2}\right) \\
H_{3} & =L_{z}^{2}+\kappa^{2} L_{y}^{2} \\
& =\left[\operatorname{sn}^{2} \beta-\operatorname{sn}^{2} \alpha\right]^{-1}\left(\operatorname{sn}^{2} \beta \partial_{\alpha}^{2}-\operatorname{sn}^{2} \alpha \partial_{\beta}^{2}\right)
\end{aligned}
$$

Hamiltonians

$$
\begin{aligned}
& =\left[\kappa^{2} \operatorname{cn}^{2} \alpha+\kappa^{\prime 2} \operatorname{cn}^{2}\left(\beta \mid \kappa^{\prime}\right)\right]^{-1}\left(\kappa^{\prime 2} \operatorname{sn}^{2}\left(\beta \mid \kappa^{\prime}\right) \partial_{\alpha}^{2}+\operatorname{dn}^{2} \alpha \partial_{\beta}^{2}\right) \\
H_{2} & =\kappa^{2} L_{x}^{2}-\kappa^{\prime 2} L_{z}^{2} \\
& =-\left[\kappa^{2} \operatorname{cn}^{2} \alpha+\kappa^{\prime 2} \operatorname{cn}^{2}\left(\beta \mid \kappa^{\prime}\right)\right]^{-1}\left(\kappa^{\prime 2} \operatorname{cn}^{2}\left(\beta \mid \kappa^{\prime}\right) \partial_{\alpha}^{2}-\kappa^{2} \operatorname{cn}^{2} \alpha \partial_{\beta}^{2}\right) \\
H_{3} & =L_{z}^{2}+\kappa^{2} L_{y}^{2} \\
& =\left[\kappa^{2} \operatorname{cn}^{2} \alpha+\kappa^{\prime 2} \operatorname{cn}^{2}\left(\beta \mid \kappa^{\prime}\right)\right]^{-1}\left(\operatorname{dn}^{2}\left(\beta \mid \kappa^{\prime}\right) \partial_{\alpha}^{2}+\kappa^{2} \operatorname{sn}^{2} \alpha \partial_{\beta}^{2}\right)
\end{aligned}
$$$$
H_{1}=L_{x}^{2}+\kappa^{\prime 2} L_{y}^{2}
$$ 
Table 2. Realizations of $S U(1,1)$ and corresponding Hamiltonians which lead to the Lamé equation.

(I):

$$
\begin{aligned}
L_{x} & =i\left(\kappa \kappa^{\prime}\left(\operatorname{sn}^{2} \beta-\operatorname{sn}^{2} \alpha\right)\right)^{-1} \\
& \times\left[\operatorname{cn} \alpha \operatorname{sn} \beta \operatorname{dn} \beta \partial_{\alpha}-\operatorname{sn} \alpha \operatorname{dn} \alpha \operatorname{cn} \beta \partial_{\beta}\right] \\
L_{y} \quad & =\left(\kappa\left(\operatorname{sn}^{2} \beta-\operatorname{sn}^{2} \alpha\right)\right)^{-1} \\
& \times\left[\operatorname{sn} \alpha \operatorname{cn} \beta \operatorname{dn} \beta \partial_{\alpha}-\operatorname{cn} \alpha \operatorname{dn} \alpha \operatorname{sn} \beta \partial_{\beta}\right] \\
L_{z} \quad & =i\left[\kappa^{\prime}\left(\operatorname{sn}^{2} \beta-\operatorname{sn}^{2} \alpha\right)\right]^{-1} \\
& \times\left[\operatorname{dn} \alpha \operatorname{sn} \beta \operatorname{cn} \beta \partial_{\alpha}-\operatorname{sn} \alpha \operatorname{cn} \alpha \operatorname{dn} \beta \partial_{\beta}\right]
\end{aligned}
$$

$S U(1,1)$ Realization

Hamiltonians

$$
\begin{aligned}
H_{1} & =\kappa^{2} L_{y}^{2}+\kappa^{2} L_{z}^{2} \\
& =\left[\operatorname{sn}^{2} \alpha-\mathrm{sn}^{2} \beta\right]^{-1}\left(\mathrm{cn}^{2} \beta \partial_{\alpha}^{2}-\mathrm{cn}^{2} \alpha \partial_{\beta}^{2}\right) \\
H_{2} & =L_{y}^{2}+\kappa^{2} L_{x}^{2} \\
& =\left[\kappa^{2}\left(\operatorname{sn}^{2} \alpha-\mathrm{sn}^{2} \beta\right)\right]^{-1}\left(\mathrm{dn}^{2} \beta \partial_{\alpha}^{2}-\mathrm{dn}^{2} \alpha \partial_{\beta}^{2}\right) \\
H_{3} & =L_{z}^{2}-\kappa^{2} L_{x}^{2} \\
& =-\left[\operatorname{sn}^{2} \alpha-\operatorname{sn}^{2} \beta\right]^{-1}\left(\operatorname{sn}^{2} \beta \partial_{\alpha}^{2}-\operatorname{sn}^{2} \alpha \partial_{\beta}^{2}\right)
\end{aligned}
$$$$
C_{2}=L_{z}^{2}-L_{x}^{2}-L_{y}^{2}=-\left[\kappa^{2}\left(\operatorname{sn}^{2} \alpha-\operatorname{sn}^{2} \beta\right)\right]^{-1}\left(\partial_{\alpha}^{2}-\partial_{\beta}^{2}\right)
$$

(II):

$$
\begin{aligned}
L_{x} & =i\left[\kappa^{2} \operatorname{cn}^{2} \alpha+\kappa^{\prime 2} \operatorname{cn}^{2}\left(\beta \mid \kappa^{\prime}\right)\right]^{-1} \\
& \times\left[-\kappa^{\prime 2} \operatorname{sn} \alpha \operatorname{sn}\left(\beta \mid \kappa^{\prime}\right) \operatorname{cn}\left(\beta \mid \kappa^{\prime}\right) \partial_{\alpha}-\operatorname{cn} \alpha \operatorname{dn} \alpha \operatorname{dn}\left(\beta \mid \kappa^{\prime}\right) \partial_{\beta}\right] \\
L_{y} \quad & =i\left[\kappa^{2} \operatorname{cn}^{2} \alpha+\kappa^{\prime 2} \operatorname{cn}^{2}\left(\beta \mid \kappa^{\prime}\right)\right]^{-1} \\
& \times\left[\operatorname{cn} \alpha \operatorname{sn}\left(\beta \mid \kappa^{\prime}\right) \operatorname{dn}\left(\beta \mid \kappa^{\prime}\right) \partial_{\alpha}-\operatorname{sn} \alpha \operatorname{dn} \alpha \operatorname{cn}\left(\beta \mid \kappa^{\prime}\right) \partial_{\beta}\right] \\
L_{z} & =\left[\kappa^{2} \operatorname{cn}^{2} \alpha+\kappa^{\prime 2} \operatorname{cn}^{2}\left(\beta \mid \kappa^{\prime}\right)\right]^{-1} \\
& \times\left[-\operatorname{dn} \alpha \operatorname{cn}\left(\beta \mid \kappa^{\prime}\right) \operatorname{dn}\left(\beta \mid \kappa^{\prime}\right) \partial_{\alpha}-\kappa^{2} \operatorname{sn} \alpha \operatorname{cn} \alpha \operatorname{sn}\left(\beta \mid \kappa^{\prime}\right) \partial_{\beta}\right] \\
C_{2} \quad & =L_{z}^{2}-L_{x}^{2}-L_{y}^{2} \\
& =\left[\kappa^{2} \operatorname{cn}^{2} \alpha+\kappa^{\prime 2} \operatorname{cn}^{2}\left(\beta \mid \kappa^{\prime}\right)\right]^{-1}\left(\partial_{\alpha}^{2}+\partial_{\beta}^{2}\right)
\end{aligned}
$$




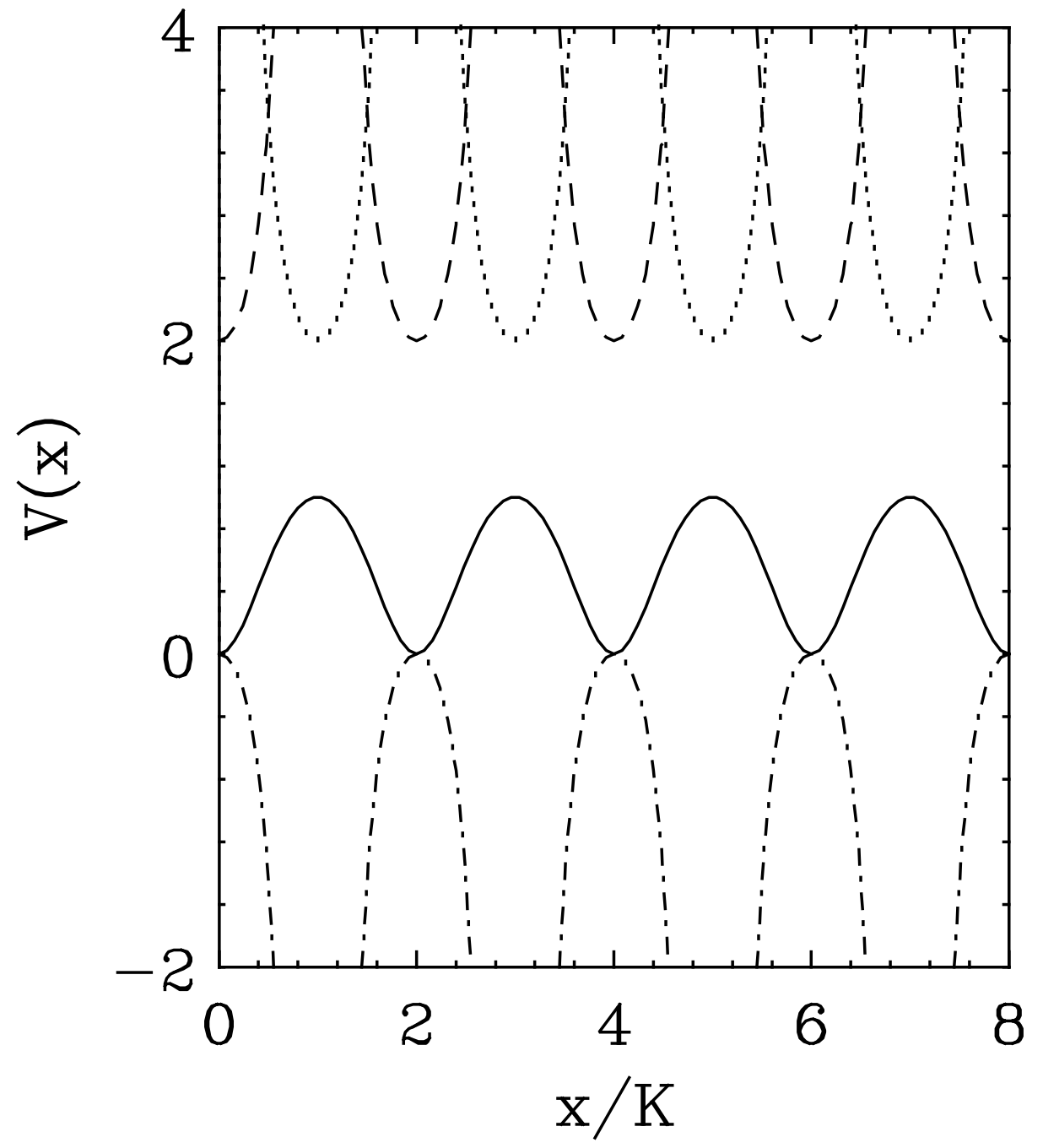


This figure "lame-fig2.jpg" is available in "jpg" format from: http://arxiv.org/ps/solv-int/9912006v1 


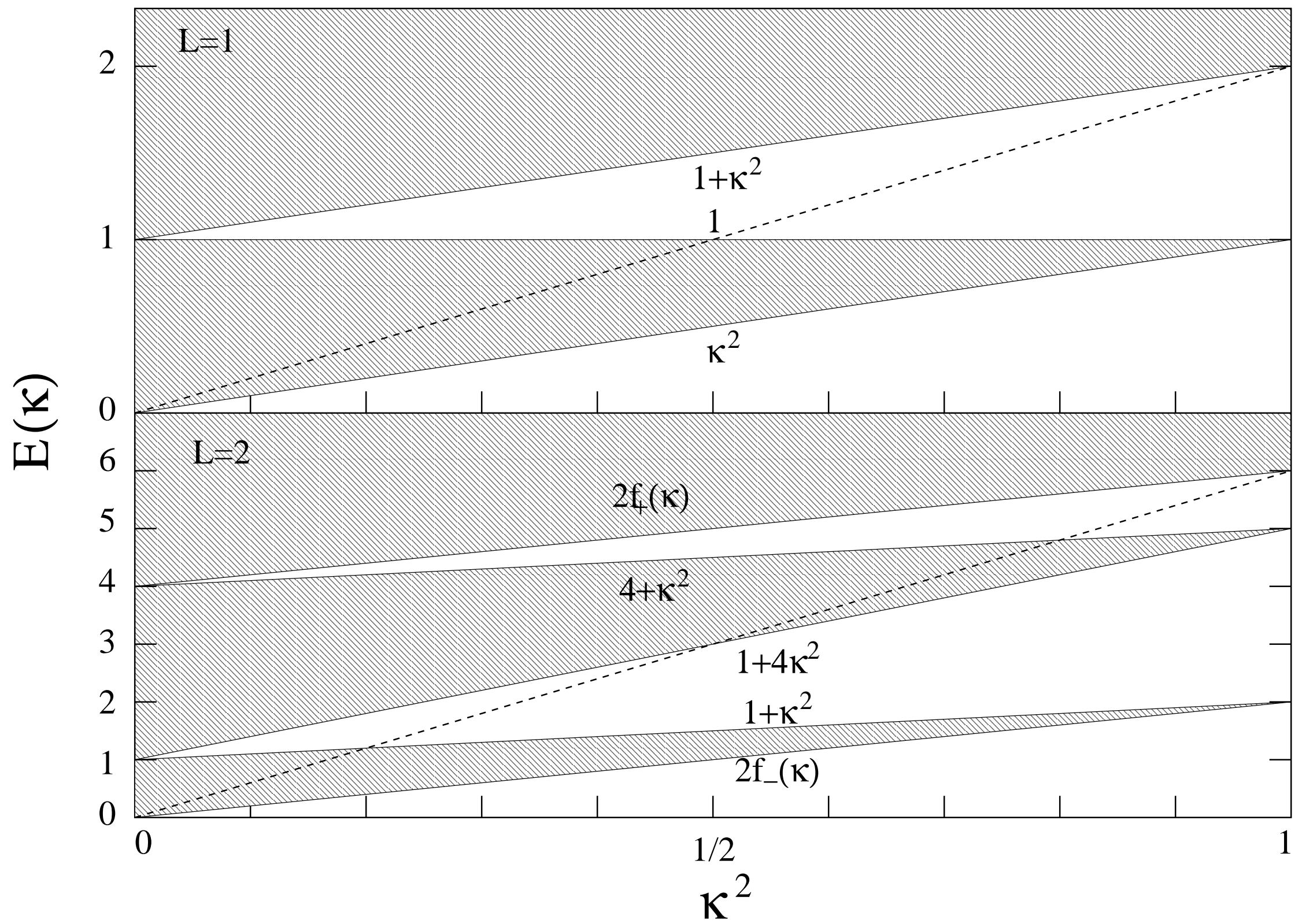


This figure "lame-fig4.jpg" is available in "jpg" format from: http://arxiv.org/ps/solv-int/9912006v1 


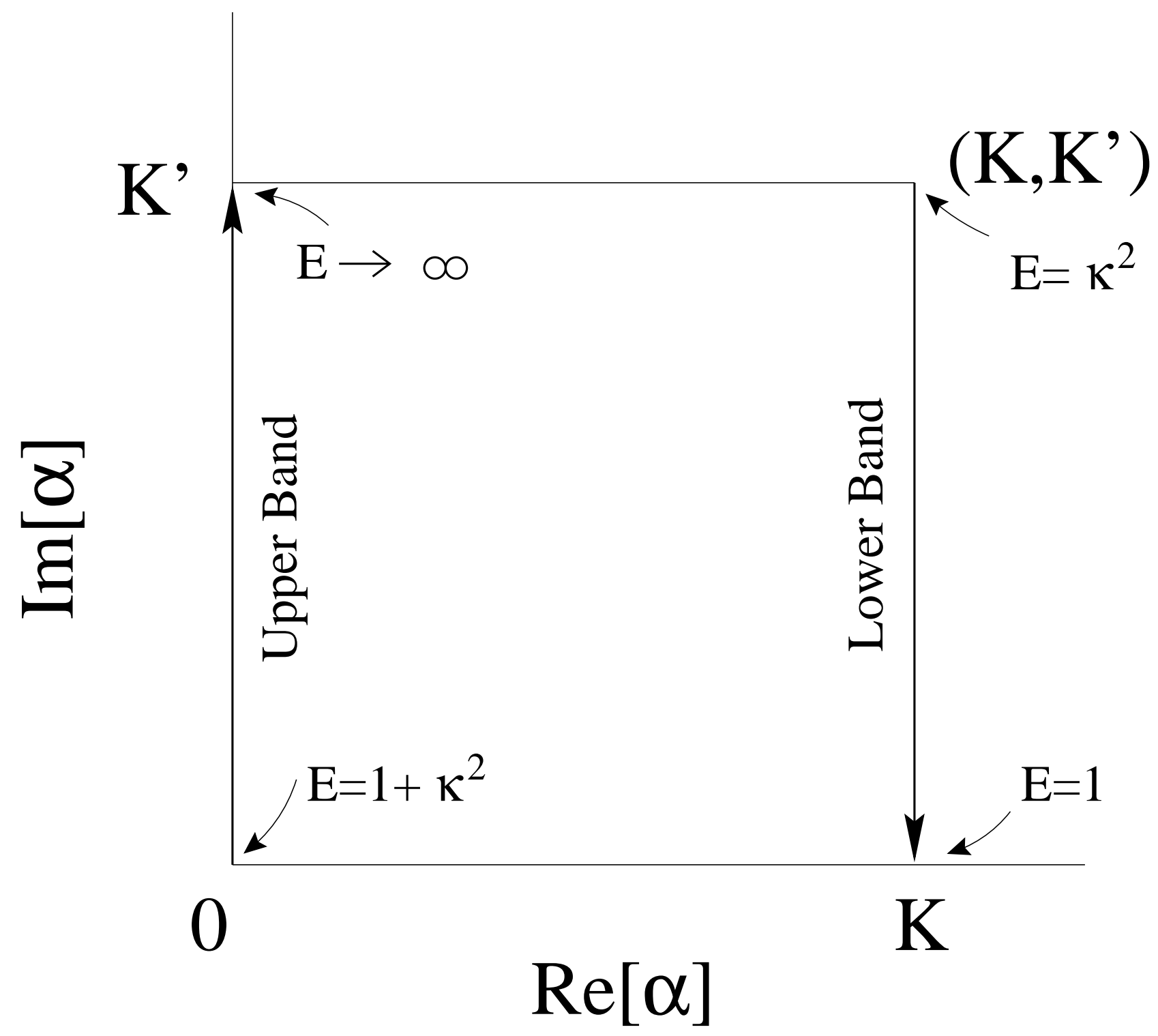




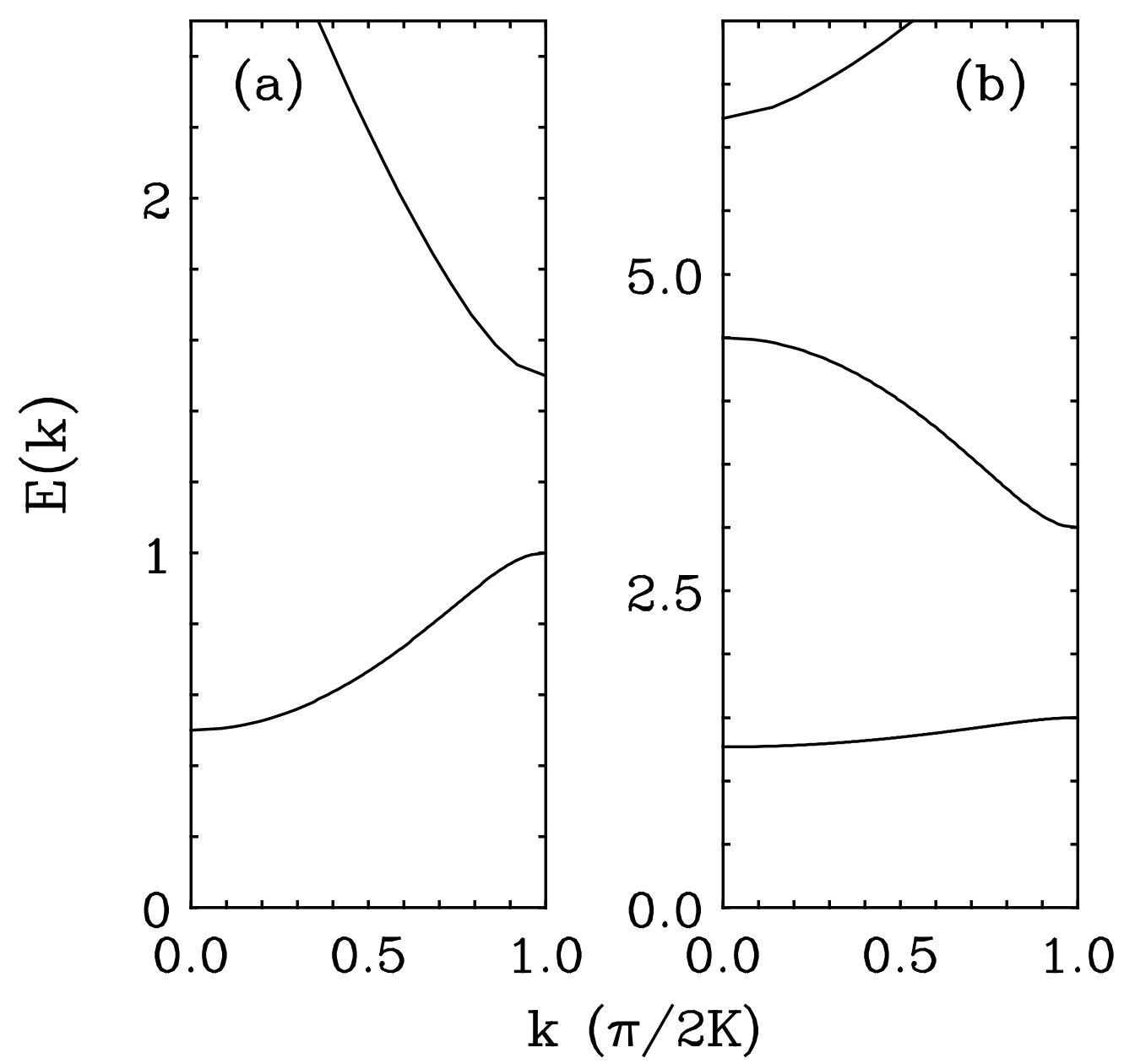




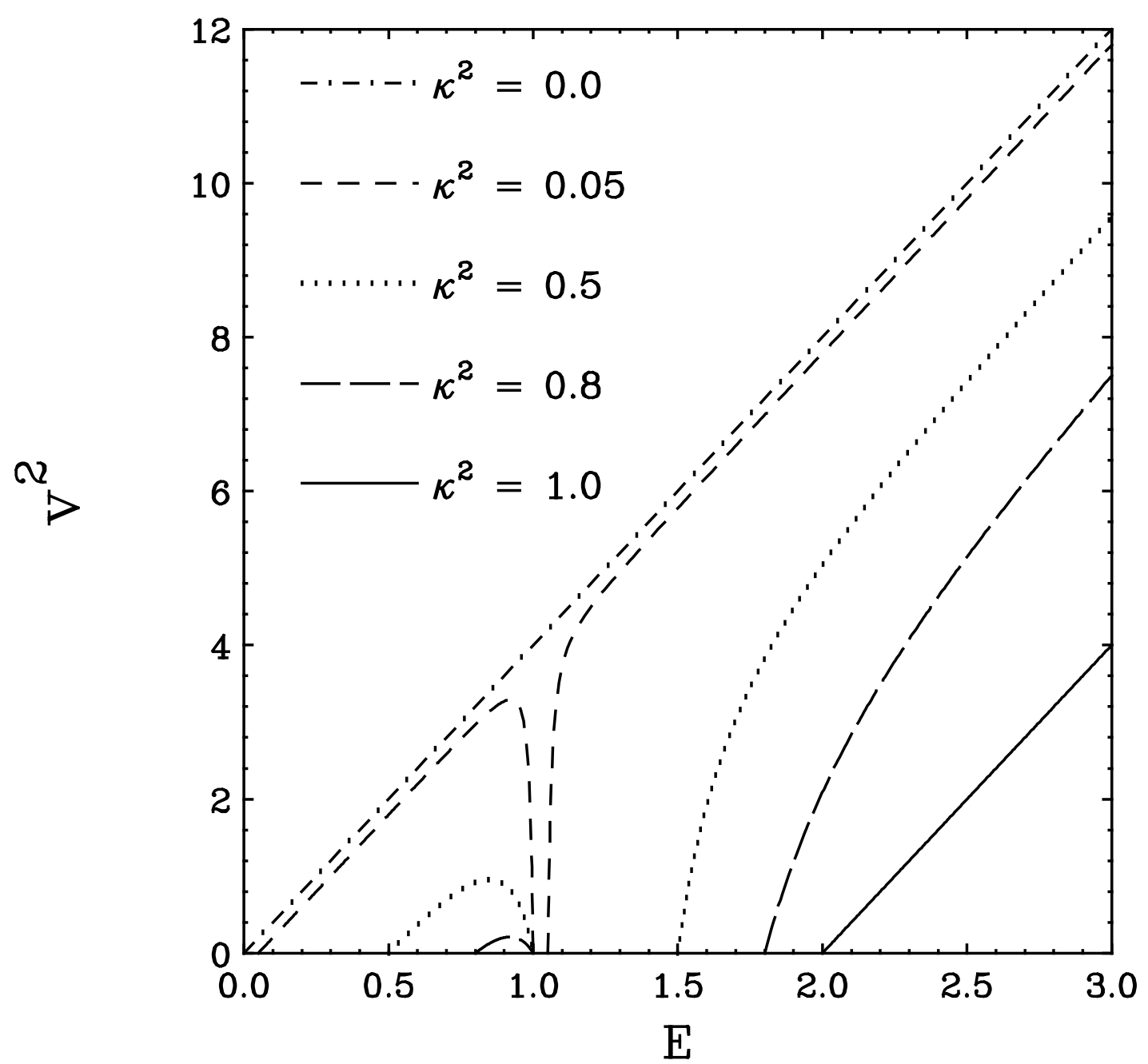




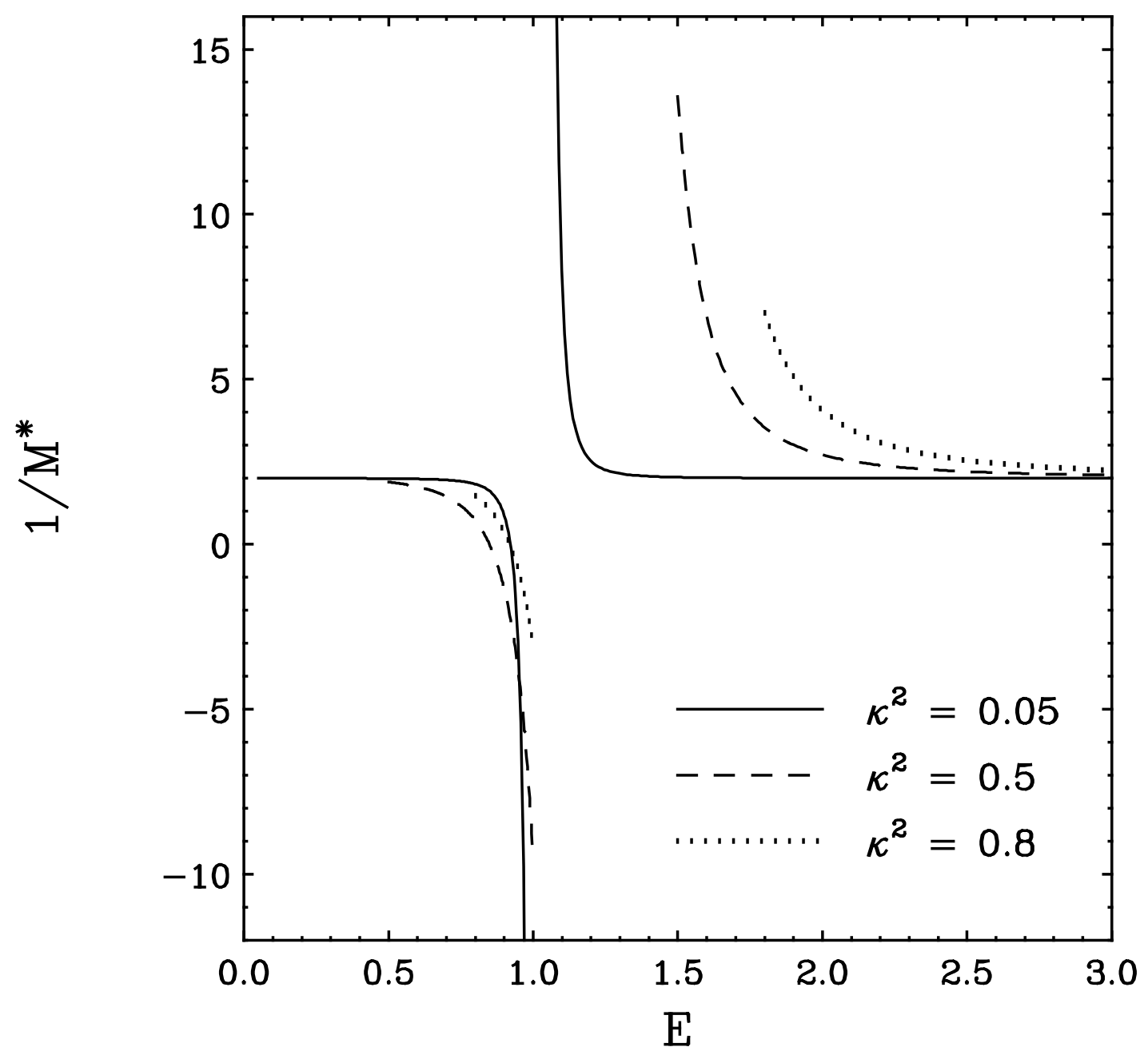

